\title{
Fiber Nonlinearity Mitigation in Optical Links That Use OFDM for Dispersion Compensation
}

\author{
Arthur J. Lowery, Senior Member, IEEE
}

\begin{abstract}
The nonlinear power limit of optical links using optical orthogonal frequency division multiplexing for dispersion compensation can be significantly improved using a simple and computationally efficient nonlinearity precompensation technique that requires no additional optical components. Simulations show that a 2-dB increase in transmission power is possible for $6 \mathrm{ps} / \mathrm{nm} / \mathrm{km}$ fibers in a 4000-km system, and this increases to $>4 \mathrm{~dB}$ for $2 \mathrm{ps} / \mathrm{nm} / \mathrm{km}$ fibers. Alternatively, the bit error ratio can be substantially reduced with precompensation when the systems are operated at an optimum power. Only a single tuning parameter is used to represent the whole link, and the system is robust against variations in this parameter.
\end{abstract}

Index Terms-Electronic dispersion compensation (EDC), fiber nonlinearity, modulation, optical communications, orthogonal frequency division multiplexing (OFDM).

\section{INTRODUCTION}

$\mathbf{E}$ LECTRONIC dispersion compensation (EDC) is becoming an attractive technology for dispersion compensation in optical links [1]. Previously, we have shown that orthogonal frequency division multiplexing (OFDM), which is widely used to adaptively compensate radio networks, could electronically compensate for dispersion in single-mode optical systems [2]. Fiber nonlinearities restricted the power per wavelength-division multiplexing (WDM) channel for 4000-km systems [3]. Nevertheless, 20-Gb/s experimental systems [4] have recently been demonstrated up to $4160 \mathrm{~km}$ [5].

Fiber nonlinearity affects all types of long-haul optical systems, unless the optical powers are kept low [6]. Low powers require frequent reamplification to maintain a sufficient signal-tonoise ratio. Thus, it is useful to try to mitigate fiber nonlinearity, as the number of optical amplifiers along a link could be reduced. Fiber nonlinearity compensation was first proposed using materials with a negative nonlinear coefficient [7], which is impracticable. Recently, nonlinearity compensation has been proposed for systems using electronic precompensation of dispersion [8]-[11], where the system is modeled but with inverse parameters for dispersion and nonlinear coefficients, then the

Manuscript received March 23, 2007; revised May 17, 2007. This work was supported in part by the Australian Research Council's Discovery Funding Scheme DP0772937.

The author is with Monash University, Clayton, VIC 3800, Australia (e-mail: arthur.lowery@eng.monash.edu.au).

Color versions of one or more of the figures in this letter are available online at http://ieeexplore.ieee.org.

Digital Object Identifier 10.1109/LPT.2007.903962 output of the model is fed into the real system using an "optical-IQ" modulator, which can modulate the laser field with a complex coefficient with inphase (I) and quadrature $(\mathrm{Q})$ components. Unfortunately, the modeling of the system requires a detailed knowledge of the dispersion map and optical power levels along the system. Real-time implementation requires extensive computation, which can only be achieved using look-up tables, rather than adaptively, as would be required in an all-optical network, for example.

This letter presents a computationally efficient technique for compensation of fiber nonlinearities in coherent optical OFDM systems [5], [12], [13]. Simple signal processing at the transmitter can be used to mitigate fiber nonlinearities, and this processing does not require exact knowledge of the dispersion map of the system, so it works over a wide range of systems. It can also be tuned with a single variable. The computation cost is similar to that used for dispersion compensation at the receiver [2], which is trivial compared with the computation cost of the Fourier transforms in OFDM. Increases in amplifier spacing of more than $15 \mathrm{~km}$ are possible for low-dispersion fibers. Higher dispersion fibers have less of an advantage, though the nonlinear power limit is already high for these fibers before compensation [3]. The key advantage of this nonlinearity compensation is that optical OFDM is now applicable to greater range of fiber dispersions.

\section{OPTICAL OFDM SYSTEM}

Fig. 1 shows the new OFDM system block diagram. The transmitter is similar to our previous work, except that a direct optical-IQ modulator [1] is used, allowing any optical amplitude and phase to be transmitted along the link. This modulator produces a single-sideband optical spectrum with a totally suppressed carrier. In-phase and quadrature components of a locally generated carrier are mixed with the optical signal at the receiver to obtain in-phase and quadrature electrical signal components [12]. These are digitized by digital-to-analog converters for processing in a standard OFDM receiver. As we have described before [2], the equalizer part of the receiver compensates for electrical phase distortion due to fiber dispersion and all electrical and optical components.

OFDM systems have very closely packed channels, so there is little walk-off due to dispersion [6] between the subcarriers' signals, as they propagate along the transmission fiber. A low walk-off causes the effects of the fiber's nonlinearity to be strong, because the subcarrier signals are phase matched along a significant proportion of the fiber's length. Precompensation of nonlinearity uses a model of an "inverse" fiber at the transmitter, so that the real transmission fiber undoes the effects 


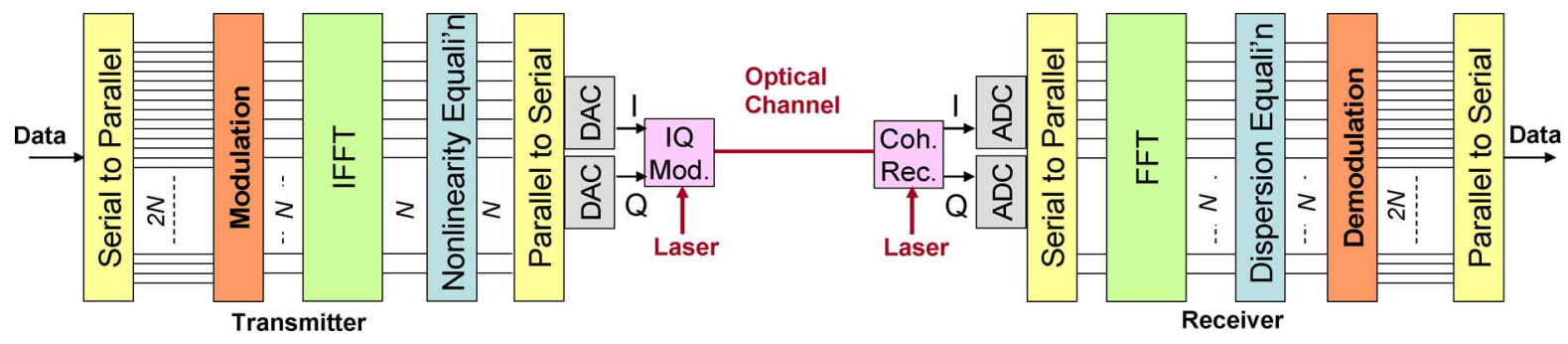

Fig. 1. Block diagram of optical OFDM with nonlinear compensation.

of this "virtual" inverse fiber. The model of the inverse fiber typically uses the split-step Fourier method [14] to simultaneously solve for the interactions between fiber dispersion and nonlinearity. This method requires the virtual fiber to be divided into short sections, typically $<1 \mathrm{~km}$, and the effects of dispersion and nonlinearity to be separately treated in each section. Because nonlinearity must be computed in the time domain, and dispersion is most efficiently computed in the frequency domain, a Fourier transform and an inverse-Fourier transform are required per section. This makes precompensation computationally expensive.

The OFDM precompensation method proposed here uses the low walk-off to an advantage because the transmission path can be approximated as dispersionless when calculating the effects of fiber nonlinearity. Neglecting dispersion greatly simplifies the nonlinear calculation; only a single step of the split-step method is required for the entire transmission path. To represent fiber nonlinearity, this calculation applies a phase advance, $\theta(t)$ in proportion to the instantaneous optical power $P(t)$, that is input to the first fiber span [15]

$$
\theta(t)=P(t) . s L_{\mathrm{eff}} \cdot 2 \pi n_{2} /\left(\lambda_{0} A_{\mathrm{eff}}\right)
$$

where $s$ is the number of fiber spans, $L_{\text {eff }}$ is an effective length of each fiber span for nonlinearity compensation, $n_{2}$ is a nonlinearity coefficient, $\lambda_{0}$ is the wavelength of the carrier, and $A_{\text {eff }}$ is the effective core area of the fiber.

This modulation can be applied either in the optical domain, where the instantaneous optical power is proportional to the square of the sum of the fields of the subcarriers, as would be detected by a photodiode. More conveniently, it can be applied in the electrical domain using a bank of hardware multipliers just after the inverse fast Fourier transform (FFT) at the transmitter. These will adjust the phase of each time sample in proportion to its magnitude squared, as its magnitude squared modulates the optical power, so (1) is implemented. Because fiber dispersion mitigates the effect of nonlinearity because of some walkoff, the effective length used here for precompensation will be less than its dispersionless value [14].

\section{Simulation Technique And EXample Constellations}

The data rate is $10 \mathrm{~Gb} / \mathrm{s}$ and the block length is 1024 bits, giving 512 OFDM carriers in an optical bandwidth of $5 \mathrm{GHz}$ with 4-quadrature amplitude modulation (QAM). The 4000-km link comprises 50 uncompensated $80-\mathrm{km}$ spans. The fiber has a loss of $0.2 \mathrm{~dB} / \mathrm{km}$, a nonlinearity coefficient, $n_{2}$, of $2.6 \times$ $10^{-20} \mathrm{~m}^{2} / \mathrm{W}$, and an effective cross section of $80 \mu \mathrm{m}^{2}$. The

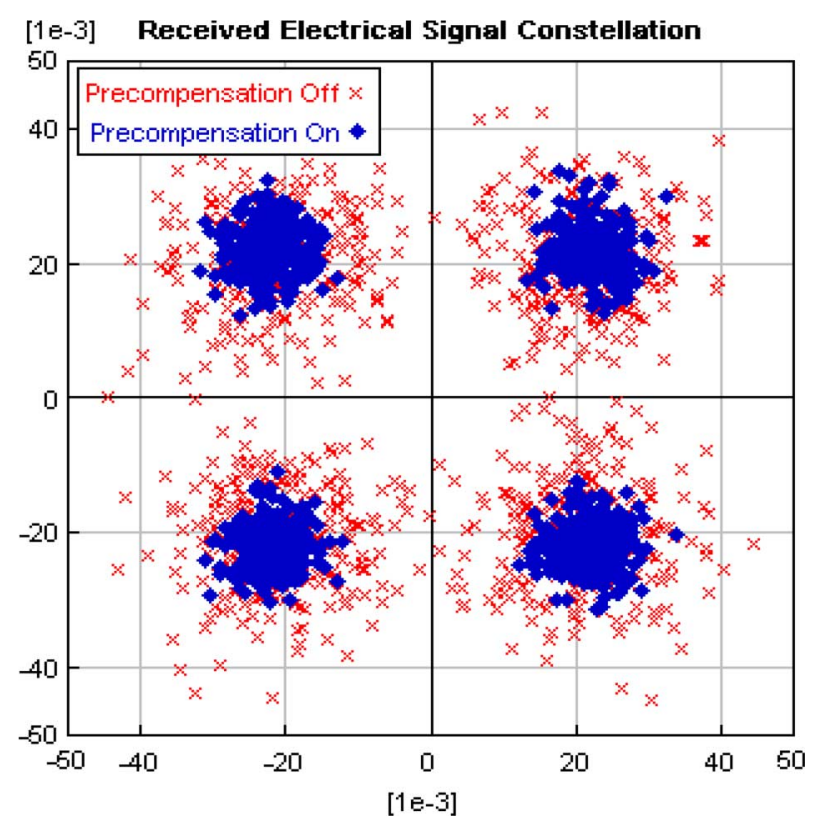

Fig. 2. Constellations with and without nonlinearity precompensation.

optical amplifiers compensate for the 16-dB fiber loss in each span, and have a noise figure (NF) of $6 \mathrm{~dB}$. The output power of each amplifier is controlled to set the input power to each $80-\mathrm{km}$ fiber span. The coherent receiver used a $10-\mathrm{mW}$ local oscillator laser and is noiseless. VPIsystems' VPItransmissionMakerWDM V7.0.1 is used for simulations with an optical bandwidth of $80 \mathrm{GHz}$ and an electrical bandwidth of $40 \mathrm{GHz}$.

Fig. 2 shows constellations for the electrical signals with and without nonlinearity compensation. Dispersion compensation is enabled in both cases. The fiber has 2-ps/nm/ $\mathrm{km}$ dispersion and the spans' input powers are $-7 \mathrm{dBm}$.

When the fiber nonlinearity is uncompensated the symbols $(x)$ in each cluster have spread into adjacent quadrants, causing errors. When considered in the frequency domain, the fiber nonlinearity acting on the instantaneous phase of the superposition of all subcarriers causes intermodulation between the subcarriers (commonly known as four-wave mixing [14]). This intermodulation perturbs the amplitude and phase of each subcarrier, spreading the points of the constellation [15], which each represent the complex-value of a single subcarrier. The bit-error ratio (BER) is estimated from the electrical signal quality measured from the constellation $q[3]$ using BER $=\frac{1}{2} \operatorname{erfc}(q / \sqrt{ } 2)$. Because of the large spread in the values of $q$, the results will be presented using $Q(\mathrm{~dB})=20 \log _{10}(q)$, without nonlinearity 
$20 \log (q), d B \quad 4000$ km 2 ps/nm/km with Amplifier Noise

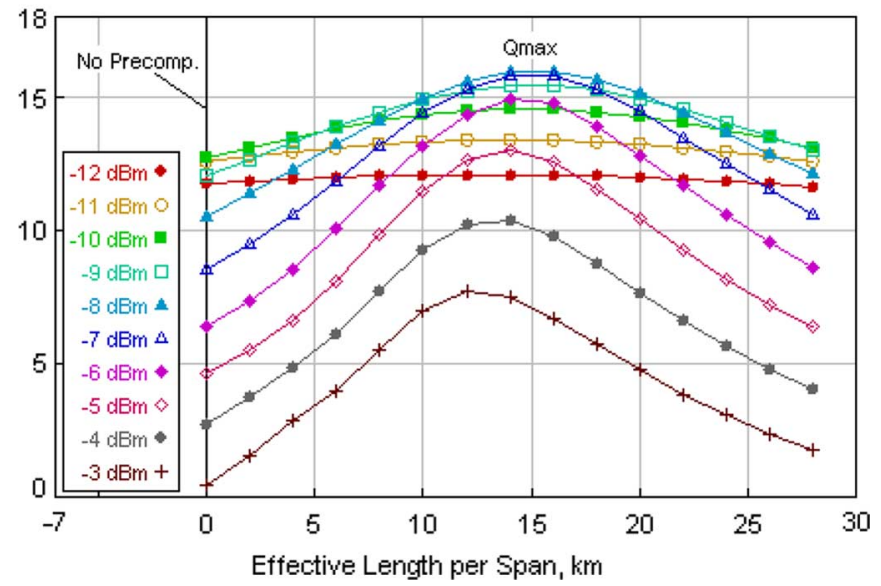

Fig. 3. Improvement in $Q=20 \log (q)$ versus effective length per span for $2 \mathrm{ps} / \mathrm{nm} / \mathrm{km}$ fiber for a range of input powers.

TABLE I

SUMMARY OF SIMULATIONS FOR THREE FIBER TYPES

\begin{tabular}{|c|c|c|c|c|c|c|}
\hline System & \multicolumn{3}{|c|}{ Uncompensated } & \multicolumn{3}{c|}{ Compensated } \\
\hline $\begin{array}{c}D, \\
\mathrm{ps} / \mathrm{nm} / \\
\mathrm{km}\end{array}$ & $\begin{array}{c}Q_{\max }, \\
\mathrm{dB}\end{array}$ & $\begin{array}{c}@ P, P, \\
\mathrm{dBm}\end{array}$ & $\begin{array}{c}\mathrm{NLT}, \\
\mathrm{dBm}\end{array}$ & $\begin{array}{c}Q_{\max }, \\
\mathrm{dB}\end{array}$ & $\begin{array}{c}\mathrm{QP}, \\
\mathrm{dBm}\end{array}$ & $\begin{array}{c}\mathrm{NLT}, \\
\mathrm{dBm}\end{array}$ \\
\hline 2 & 12.7 & -10 & $>-8$ & 15.9 & -8 & $>-4$ \\
\hline 6 & 13.1 & -10 & $>-8$ & 14.3 & -9 & $>-6$ \\
\hline 16 & 13.8 & -9 & $>-7$ & 14.5 & -9 & $>-6$ \\
\hline
\end{tabular}

compensation $Q=8.5 \mathrm{~dB}$ giving a BER of 0.004 . Precompensating the nonlinearity with an effective length per span of $15 \mathrm{~km}$ maximizes $Q$ for this input power and dispersion. The clusters

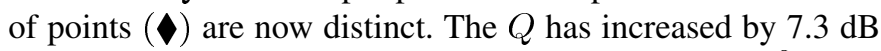
to $15.8 \mathrm{~dB}$, which is sufficient to achieve a BER $<10^{-9}$.

\section{ACHIEVABLE IMPROVEMENTS}

Fig. 3 plots the $Q$ versus the effective length for a set of input powers and $2 \mathrm{ps} / \mathrm{nm} / \mathrm{km}$ fiber. The effective length is used to tune the nonlinear compensation. It is clear that for every input power the $Q$ improves significantly for nonzero effective lengths, except for very low input powers $(<11 \mathrm{dBm})$ where amplifier noise becomes the limiting factor. For systems away from the noise limit ( $>-6 \mathrm{dBm}$ ), the increase in $Q$ is $>7 \mathrm{~dB}$.

Table I summarizes results for three types of fibers with different dispersions $D$. For systems design, $Q_{\max }$ is a good measure of the optimum performance that can be obtained by tuning the effective length; a conservative estimate of input power, $P$, at this optimum is given, noting that results were obtained for 1-dB steps. Without compensation, low dispersion fibers suffer the greatest $Q$ penalties due to nonlinearity and thus require the lowest input powers [3]. Conversely, with precompensation, low-dispersion fibers offer the highest $Q$. This is because the precompensation is more effective when the walk-off along the fiber is low.

Precompensation also allows systems to be operated at higher powers, for example, at the beginning of their lives. Defining nonlinear threshold (NLT) as the power limit for the $Q$ to be
$>10 \mathrm{~dB}$, the NLT increases by $>1 \mathrm{~dB}$ for standard fibers and by $>4 \mathrm{~dB}$ for low-dispersion fibers.

\section{CONCLUSION}

A simple modification to coherent optical OFDM has been proposed that mitigates the effects of nonlinearity in the transmission path, particularly for low-dispersion fibers. This requires a simple computation for each time sample in the transmitter's waveform. With compensation, the nonlinear limits for near-zero and reduced dispersion fibers are increased to above that for standard single-mode fibers (provided large-core areas are used for each type of fiber). A single tuning parameter is used so that little knowledge is required of the actual fiber plant to achieve a reasonable benefit.

The results presented are for a single optical channel, rather than a WDM OFDM system. However, as the dominant effect of fiber nonlinearity is the interaction between the closely spaced subcarriers (approximately $10 \mathrm{MHz}$ ), rather than the widely spaced WDM channels ( $>10 \mathrm{GHz}$ spacing), this method is likely to also give significant improvements in OFDM WDM systems.

\section{REFERENCES}

[1] J. McNicol, M. O'Sullivan, K. Roberts, A. Comeau, D. McGhan, and L. Strawczynski, "Electrical domain compensation of optical dispersion," in Tech. Dig. Conf. Optical Fiber Communications, Anaheim, CA, 2005, vol. 5, pp. 269-271.

[2] A. J. Lowery and J. Armstrong, "Orthogonal frequency division multiplexing for dispersion compensation of long-haul optical systems," Opt. Express, vol. 14, pp. 2079-2084, 2006.

[3] A. J. Lowery, L. B. Du, and J. Armstrong, "Performance of optical OFDM in ultralong-haul WDM lightwave systems," J. Lightw. Technol., vol. 25, no. 1, pp. 131-138, Jan. 2007.

[4] B. J. C. Schmidt, A. J. Lowery, and J. Armstrong, "Experimental demonstrations of $20 \mathrm{Gbit} / \mathrm{s}$ direct-detection optical OFDM and 12 Gbit/s with a colorless transmitter," presented at the Conf. Optical Fiber Communications, Anaheim, CA, 2007, Paper PDP18.

[5] S. L. Jansen, I. Morita, N. Tadeka, and H. Tanaka, "20-Gb/s OFDM transmission over 4,160-km SSMF enabled by RF-pilot tone phase noise compensation," presented at the Conf. Optical Fiber Telecommunications, Anaheim, CA, 2007, Paper PDP15.

[6] T. Li and I. Kaminow, Eds., Optical Fiber Telecommunications IVB. San Diego, CA: Academic, 2002, ch. 13.

[7] C. Paré, A. Villeneuve, P.-A. Belanger, and N. J. Doran, "Compensating for dispersion and the nonlinear Kerr effect without phase conjugation," Opt. Lett., vol. 21, pp. 459-461, 1996.

[8] R. I. Killey, P. M. Watts, V. Mikhailov, M. Glick, and P. Bayval, "Electronic dispersion compensation by signal predistortion using digital processing and a dual-drive Mach-Zehnder modulator," IEEE Photon. Technol. Lett., vol. 17, no. 3, pp. 714-716, Mar. 2005.

[9] R. I. Killey, "Dispersion and nonlinearity compensation using electronic predistortion techniques," in Proc. IEE Seminar on Fibre Communications Electronic Signal Processing 2005-11310, Dec. 15, 2005, 0537-9989, 0-14-2/6.

[10] R. J. Essiambre, P. J. Winzer, W. X. Qing, W. Lee, C. A. White, and E. C. Burrows, "Electronic predistortion and fiber nonlinearity," IEEE Photon. Technol. Lett., vol. 18, no. 17, pp. 1804-1806, Sep. 1, 2006.

[11] K. Roberts, C. Li, L. Strawczynski, and M. O'Sullivan, "Electronic precompensation of optical nonlinearity," IEEE Photon. Technol. Lett., vol. 18, no. 1, pp. 403-405, Jan. 1, 2006.

[12] W. Shieh and C. Athaudage, "Coherent optical orthogonal frequency division multiplexing," Electron. Lett., vol. 42, pp. 587-588, 2006.

[13] W. Shieh, X. Yi, and Y. Tang, "Transmission experiment of multi-gigabit coherent optical OFDM systems over $1000 \mathrm{~km}$ SSMF fibre," Electron. Lett., vol. 43, no. 3, pp. 183-184, 2007.

[14] G. P. Agrawal, Nonlinear Fiber Optics, 2nd ed. San Diego, CA: Academic, 1995.

[15] J. P. Gordon and L. F. Mollenauer, "Phase noise in photonic communications systems using linear amplifiers," Opt. Lett., vol. 15, no. 23, pp. 1351-1353, , 1990. 